\title{
Packing fraction of particles with a Weibull size distribution
}

\author{
H. J. H. Brouwers \\ Department of the Built Environment, Eindhoven University of Technology, P.O. Box 513, 5600 MB Eindhoven, The Netherlands
}

(Received 23 February 2016; revised manuscript received 31 May 2016; published 13 July 2016)

\begin{abstract}
This paper addresses the void fraction of polydisperse particles with a Weibull (or Rosin-Rammler) size distribution. It is demonstrated that the governing parameters of this distribution can be uniquely related to those of the lognormal distribution. Hence, an existing closed-form expression that predicts the void fraction of particles with a lognormal size distribution can be transformed into an expression for Weibull distributions. Both expressions contain the contraction coefficient $\beta$. Likewise the monosized void fraction $\varphi_{1}$, it is a physical parameter which depends on the particles' shape and their state of compaction only. Based on a consideration of the scaled binary void contraction, a linear relation for $\left(1-\varphi_{1}\right) \beta$ as function of $\varphi_{1}$ is proposed, with proportionality constant $B$, depending on the state of compaction only. This is validated using computational and experimental packing data concerning random close and random loose packing arrangements. Finally, using this $\beta$, the closed-form analytical expression governing the void fraction of Weibull distributions is thoroughly compared with empirical data reported in the literature, and good agreement is found. Furthermore, the present analysis yields an algebraic equation relating the void fraction of monosized particles at different compaction states. This expression appears to be in good agreement with a broad collection of random close and random loose packing data.
\end{abstract}

DOI: 10.1103/PhysRevE.94.012905

\section{INTRODUCTION}

Weibull (or Rosin-Rammler) distributions [1-3] are useful for representing particle size distributions generated by fragmentation processes in powder technology, cementitious materials, coal, soil physics, nuclear physics, food, astrophysics, genetics, sprays, fuel combustion, brittle fracture, and geology [3-6]. The random (or disordered) packing of solid particle assemblies is, among others, relevant to physicists, biologists, and engineers, and is a long-standing problem in mathematics. There is practical as well as fundamental interest in understanding the relationship between the particle shape and particle size distribution on the one hand, and packing fraction on the other.

The void fraction $\varphi_{1}$ (i.e., unity minus packing fraction $f_{1}$ ) of monosized, that is, identical, particles depends on their shape and method of packing: ordered (crystalline) or disordered (amorphous), where the latter furthermore depends on the densification: the density of the final assembly lies between the so-called "random loose packing limit" (RLP) and "random close packing limit" (RCP). For a number of particle shapes, both RLP and RCP measured void (or packing) fraction values have been reported $[7,8]$. These two limits have been extensively studied, but establishing their exact definitions has been a difficult challenge [9]. Mathematically, the RCP state is difficult to define because by introducing order, higher packing fractions can be obtained. A more recent concept that has been suggested to replace RCP is that of the maximally random jammed (MRJ) state. According to some well-defined order metrics, this state corresponds to the most

Published by the American Physical Society under the terms of the Creative Commons Attribution 3.0 License. Further distribution of this work must maintain attribution to the author(s) and the published article's title, journal citation, and DOI. disordered among all jammed (mechanically stable) packings, and has first been studied for spherical, and subsequently for nonspherical, particles [9-13]. The concept of the random close packing limit has been synonymous with the idea of a densest random packing and is found to lie at around 0.64 for spheres. Random loose packing (RLP) is considered as the loosest way particles can pack, and it is recognized that friction and cohesion play a key role. For cohesionless particles with friction, a reproducible packing fraction is found [7,8,14-18], with 0.54 as a generally accepted value for the lower limit of random monosized sphere packings. These reproducible values indicate that RLP and RCP correspond to well-defined "geometrical structures," yielding the perception that random particle packings should have a common structure.

Another complication arises when ordered or disordered arrangements contain similar (isomorphous) particles of different sizes that are randomly placed. For mixes of discretely sized particles and continuous power-law and lognormal distributions, analytical expressions for the void fraction have been derived [19-23]. In Sec. II, the parameters governing the Weibull and the lognormal (or log-normal) distributions are related to each other. Then, based on the available void fraction of the lognormal distribution [23], a closed-form expression is derived for the void fraction of the Weibull distribution.

Besides depending on the monosized void fraction $\varphi_{1}$ of the considered particle shape and the distribution characteristics, these lognormal and Weibull packing fraction expressions also contain a contraction coefficient $\beta$. This is a physically defined parameter, likewise the monosized void fraction value, and it only depends on the particle shape and the mode of compaction (between RLP and RCP) only. To bring this physical parameter into a consistent pattern, in Sec. III, the magnitude and nature of this contraction coefficient are analyzed in more detail, and an algebraic expression relating it to the monosized void fraction is established. In Sec. IV, this analysis of $\beta$ enables an extensive validation of the derived Weibull void fraction expression using a broad collection of available empirical 
data, yielding good agreement. Finally, the Appendix presents an analysis that yields a general relation between the void fractions at different packing states of monosized particles. This expression is validated for the random loose and close void fractions using computational and empirical data of a broad collection of particle types.

\section{RELATING WEIBULL AND LOGNORMAL SIZE DITRIBUTIONS}

In this section the parameters governing the Weibull and lognormal distributions are uniquely related to each other. Subsequently, the closed-form expression for the void fraction of lognormal size distributions [23] is transformed into an expression for Weibull distributions.

The cumulative distribution function (cumulative finer fraction) of the Weibull or Rosin-Rammler distribution [1-4] reads

$$
F(d)=1-\exp \left(-\left[\frac{d}{\delta}\right]^{m}\right) .
$$

Note that for $m=1$ it corresponds to the exponential distribution. The cumulative distribution function of the lognormal distribution reads $[3,23]$

$$
F(d)=\frac{1}{\sqrt{2 \pi} \ln \sigma_{g}} \int_{-\infty}^{d} \exp \left[-\frac{\left(\ln d-\ln d_{g}\right)^{2}}{2 \ln ^{2} \sigma_{g}}\right] d(\ln d),
$$

with $d_{g}$ (or $d_{0.5}$ as geometric mean size of the distribution, $\sigma_{g}$ as the geometric standard deviation, and $\ln \sigma_{g}$ as the standard deviation.

The spread of the Weibull distribution is governed by the shape parameter $m$, which has a similar role as the standard deviation $\ln \sigma_{g}$ in the lognormal distribution, as both $m$ and $\ln \sigma_{g}$ define the spread of both distributions. The parameter $\delta$, likewise $d_{g}$, is a scaling parameter, which does not affect the packing fraction. Their variation merely implies a multiplication of the size of all particles with the same factor.

In order to characterize and match the spread of both distributions, which govern their packing fraction, the size ratio $d_{x} / d_{1-x}$ of each distribution is equated, whereby

$$
d_{x}=F^{-1}(x)
$$

with $F^{-1}$ as the inverse cumulative distribution function or quantile function. The quantile function of Eq. (1) reads

$$
F^{-1}(x)=\delta[-\ln (1-x)]^{1 / m} .
$$

For the spread of the Weibull distribution it therefore holds that

$$
\frac{d_{x}}{d_{1-x}}=\left[\frac{\ln (x)}{\ln (1-x)}\right]^{1 / m} .
$$

The quantile function of Eq. (2) reads

$$
F^{-1}(x)=d_{g} \exp \left[\sqrt{2} \ln \sigma_{g} \operatorname{erf}^{-1}(2 x-1)\right] ;
$$

see Eq. (3), with $\operatorname{erf}^{-1}$ as inverse error function. As $\operatorname{erf}^{-1}(-x)=-\operatorname{erf}^{-1}(x)$ it follows that for the lognormal distribution it holds that

$$
\frac{d_{x}}{d_{1-x}}=e^{-2 \operatorname{erf}^{-1}(2 x-1) \sqrt{2} \ln \sigma_{g}} .
$$

TABLE I. Proportionality coefficient, $\alpha$, computed for various spreads of the lognormal and Weibull distributions.

\begin{tabular}{llcc}
\hline \hline$x$ & $2 x-1$ & $\operatorname{erf}^{-1}(2 x-1)$ & $\alpha$ [Eq. (8)] \\
\hline 0.9 & 0.8 & 0.906 & 1.702 \\
0.95 & 0.9 & 1.163 & 1.748 \\
0.99 & 0.98 & 1.645 & 1.862 \\
0.999 & 0.998 & 2.186 & 2.022 \\
\hline \hline
\end{tabular}

Equating Eqs. (5) and (7) yields

$$
\ln \sigma_{g}=\frac{\ln \left[\frac{\ln (x)}{\ln (1-x)}\right]}{-2 m \sqrt{2} \operatorname{erf}^{-1}(2 x-1)}=\frac{\alpha}{m \sqrt{2}} .
$$

So $m$ and $\ln \sigma_{g}$ are inversely proportional. For $x=$ $0.9,0.95,0.99$, and 0.999 , in Table I the computed proportionality constant $\alpha$ are given, which lie in a relatively narrow range. So, for these computed $\alpha$, given an $m$ of the Weibull distribution, the corresponding $\ln \sigma_{g}$ of the matching lognormal distribution can be computed.

In order to match both distributions, the $d_{0.5}\left[\mathrm{~F}^{-1}(0.5)\right]$ of both distributions are also equated using Eqs. (4) and (6), invoking $\operatorname{erf}^{-1}(0)=0$ in the latter, yielding

$$
d_{g}=\delta(\ln 2)^{1 / m}
$$

or

$$
\ln d_{g}=\ln \delta+\frac{\ln (\ln 2)}{m} .
$$

One can see that relating the spreads of the distributions leads to a relation between the shape parameter $m$ and $\ln \sigma_{g}$, and that relating the scale parameters yields a relation between $\delta$ and $d_{g}$, which are linearly proportional.

Using Eqs. (8) and (10), the relative difference of Weibull and lognormal distributions are evaluated for the $\alpha$ range listed in Table I, yielding $\alpha \approx 2$ as a coefficient that matches both distributions best (highest $R^{2}$ ) over a wide range of $m$ and $\ln \sigma_{g}$, and which is used henceforth.

In Fig. 1 two lognormal and Weibull distributions are presented, whereby their parameters are coupled, based on the equations above. One can see that for the largest $m[m=4 \sqrt{2}$, corresponding to $\ln \sigma_{g}=0.25$; see Eq. (8)], indicated by (b), both distributions are very similar.

Now that both distributions are uniquely related, also the void fraction of particles with a Weibull distribution can be assessed using the closed-form expression of the lognormal distribution [23]:

$$
\varphi^{\mathrm{LN}}=\varphi_{1} e^{-\sqrt{2 \pi}\left(1-\varphi_{1}\right) \beta \ln \sigma_{g}}=\varphi_{1} \sigma_{g}^{-\sqrt{2 \pi}\left(1-\varphi_{1}\right) \beta} .
$$

This equation features that the void fraction becomes the monosized void fraction $\varphi_{1}$ for $\ln \sigma_{g}=0$. The void fraction of the Weibull distribution follows by combining Eqs. (8) and (11) and using $\alpha=2$, producing

$$
\varphi^{\mathrm{WB}}=\varphi_{1} e^{-2 \sqrt{\pi}\left(1-\varphi_{1}\right) \beta / m} .
$$

Equation (12) provides the void fraction of a continuous Weibull distribution, which depends on the void fraction of the single-sized particles $\left(\varphi_{1}\right)$, the distribution shape parameter 


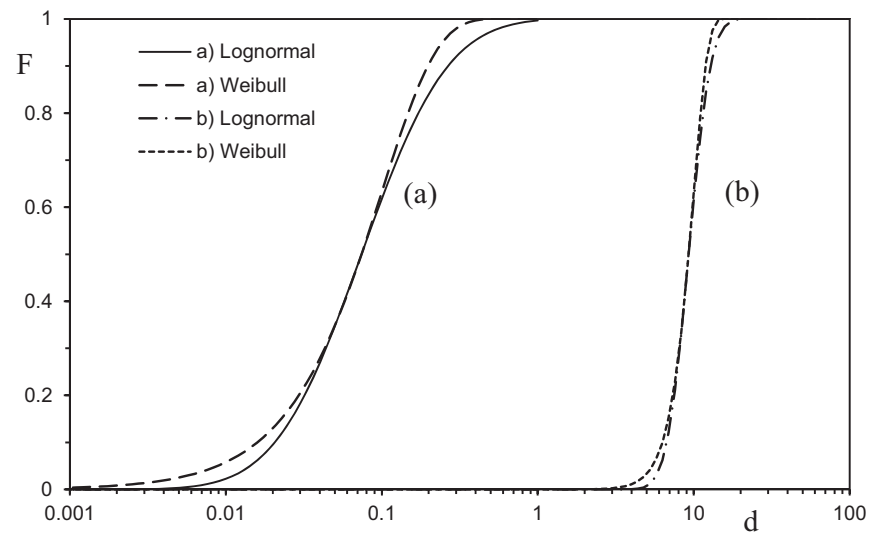

FIG. 1. The cumulative finer distribution $F$ of two lognormal and Weibull distributions as a function of the particle size $d$ : (a) $\mathrm{m}=\sqrt{2}, \ln \sigma_{g}=1, \delta=0.1, \ln d_{g}=-2.56$, and (b) $\mathrm{m}=4 \sqrt{2}, \ln \sigma_{g}=$ $0.25, \delta=10, \ln d_{g}=2.24$, whereby $m$ and $\ln \sigma_{g}$ on the one hand, and $\ln \delta$ and $\ln d_{g}$ on the other, are coupled by Eqs. (8) and (10), respectively (with $\alpha=2$ ).

$m$, and the contraction coefficient $(\beta)$, which are all specified properties.

The scaling parameter $\delta$ does not feature in Eq. (12), indeed, because its variation only implies that all particles' sizes are multiplied with the same factor. As void fractions are determined by relative particle sizes, this does not affect the void fraction. Equation (12) furthermore indicates that the void fraction of the system tends to the monosized void fraction when the shape parameter $m$ tends to infinity, i.e., the distribution tending to a monosized distribution, as would be expected (see also Fig. 1).

The void fraction expressions (11) and (12) contain a contraction coefficient $\beta$. So, besides depending on the monosized void fraction $\varphi_{1}$ of the considered particle shape and the distribution characteristics $\left(\ln \sigma_{g}\right.$ and $m$ ), these expressions also contain this contraction coefficient. This is a physically defined parameter, likewise the monosized void fraction value $\varphi_{1}$. And like the monosized void fraction, $\beta$ only depends on the particle shape and the mode of compaction (between loose and close) only. For spheres, from RLP to RCP, its magnitude is known [21]. For other particle types, however, information is scarce. In the next section the magnitude and nature of the contraction coefficient are therefore analyzed in more detail.

\section{CONTRACTION COEFFCIENT}

Compared to an arrangement of randomly packed identical (monosized) particles, a polydisperse disordered packing features a lower void fraction. For the understanding of the associated void contraction coefficient $\beta$, a consideration of a binary packing, i.e., consisting of two discretely sized isomorphous particle classes, provides fundamental information.

\section{A. Void fraction of binary particle distributions}

Binary mixes of similarly shaped particles attain a higher packing fraction, or lower void fraction, than the monosized value of large or small constituents individually. For the study of void change in the case of polydispersity, the binary void

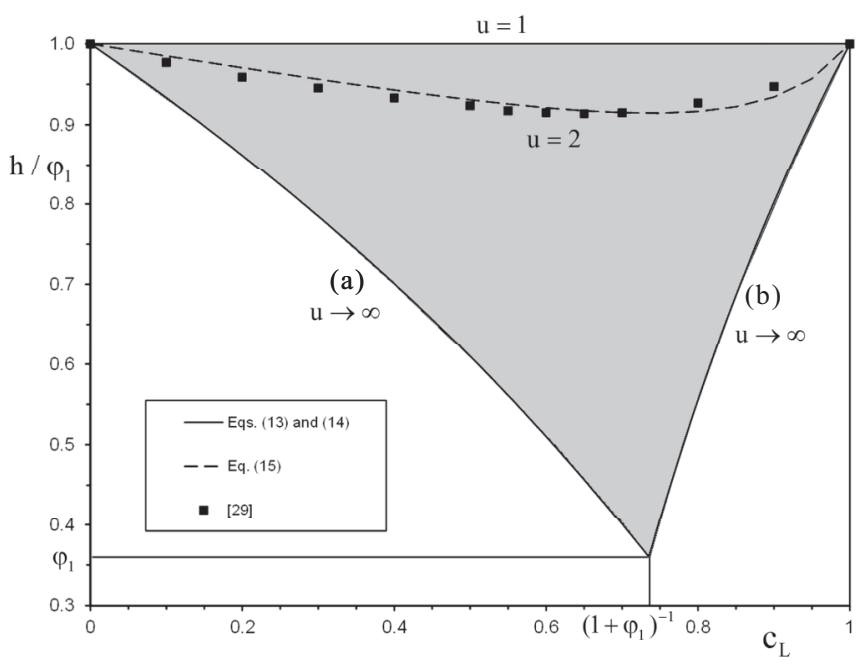

FIG. 2. Void fraction reduction quotient $\left(h / \varphi_{1}\right)$ of random close packing (RCP) of binary spheres (shaded area) as a function of the large sphere volume fraction $c_{L}\left(0 \leqslant c_{L} \leqslant 1\right)$ and sphere size ratio $u(1 \leqslant u<\infty)$. The boundaries for $u \rightarrow \infty$ follow from Eqs. (13) and (14) with $\varphi_{1}=0.36$ [indicated by (a) and (b), respectively]. The approximation [Eq. (15) with $\beta=0.20, \varphi_{1}=0.36$ ] and the simulation results of [29] for $u=2$ are included as well. For $u \rightarrow \infty$ the composition $\left[c_{L}=\left(1+\varphi_{1}\right)^{-1}\right]$ and the scaled void fraction $\left(h / \varphi_{1}=\varphi_{1}\right)$ pertaining to maximum void reduction are indicated.

fraction graph, as first constructed by Furnas [24,25], and reconstructed in [20], is instrumental. In Fig. 2, as an example, the void fraction graph of a random close packing (RCP) of binary spheres is presented as a function of the large sphere volume fraction $c_{L}$ and size ratio $u$ (ratio of the large and small sphere diameters).

The figure actually features the binary void fraction $h\left(c_{L}, u\right)$, divided (scaled) by the monosized void fraction $\varphi_{1}$. This scaled binary void fraction, $h / \varphi_{1}$, governs the void fraction reduction and was instrumental in deriving the void fraction of discretely distributed particle packings [19,20,23] and continuous particle size distributions [19,23], and $\beta$ appears in closed-form expressions for them. For the shown particular RCP of spheres it holds that $\varphi_{1} \approx 0.36[7,21]$. One can see in Fig. 2 that for size ratio $u=1$, the scaled void fraction, $h / \varphi_{1}$, is unity in the entire compositional range; i.e., the binary void fraction corresponds to a monosized void fraction. Also for an arrangement of small $\left(c_{L}=0\right)$ or large $\left(c_{L}=1\right)$ spheres only, obviously $h / \varphi_{1}$ is unity. For $0<c_{L}<1$ and $u>1$, the void fraction is reduced (packing fraction increased) by combining the two discretely sized particles, reflected in $h / \varphi_{1}<1$. This concept and the same binary graphs can also be constructed for combinations of similar nonspherical particles; $u$ is then the ratio of a characteristic size of the two similar particles.

In this graph two more situations are included, namely, a size ratio close to unity and an infinitely large size ratio. Furnas [24,25] introduced the concept of noninteracting particle classes, i.e., particle groups where the particles of the larger size class are much larger than that of the small size class particles. Hence, by combining two noninteracting size groups, i.e., for $u \rightarrow \infty$, one obtains as resulting void fractions 
$[23,26-28]$,

$$
\begin{gathered}
h\left(c_{L}, u \rightarrow \infty\right)=\frac{\left(1-c_{L}\right) \varphi_{1}}{1-c_{L} \varphi_{1}}\left[0 \leqslant c_{L} \leqslant\left(1+\varphi_{1}\right)^{-1}\right], \\
h\left(c_{L}, u \rightarrow \infty\right)=\frac{c_{L}-1+\varphi_{1}}{c_{L}}\left[\left(1+\varphi_{1}\right)^{-1} \leqslant c_{L} \leqslant 1\right] .
\end{gathered}
$$

Both equations are shown in Fig. 2, using $\varphi_{1}=0.36$; for convenience Eqs. (13) and (14) are termed (a) and (b), respectively. The maximum scaled void reduction occurs at their intersection, composition $c_{L}=\left(1+\varphi_{1}\right)^{-1} \approx 0.26$, and the maximum scaled reduction then amounts to $\varphi_{1}$. So, whereas a monosized packing, composed of small $\left(c_{L}=0\right)$ or large $\left(c_{L}=1\right)$ particles only, possesses void fraction $\varphi_{1}$, this minimum binary void fraction $h$ takes a value of $\varphi_{1}^{2}$. This particular packing was referred to by Furnas [24,25] as "saturated." For all $c_{L}$ and $u$, the scaled void fraction is enclosed by Eqs. (13) and (14), and the line $h / \varphi_{1}=1$ (pertaining to $u=1$ ), indicated in Fig. 2 by the shaded area.

For small $u-1$, the binary void fraction can be expressed as $[19,21]$

$$
\begin{aligned}
& h\left(c_{L}, u\right) \\
& =\frac{\varphi_{1}\left[c_{L}\left(1-u^{3}\right)+u^{3}\right]}{c_{L}\left(1-u^{3}\right)+u^{3}+\frac{4}{3} \beta\left(1-\varphi_{1}\right)\left(1-c_{L}\right) c_{L}\left(u^{3}-1\right)},
\end{aligned}
$$

which for $u \downarrow 1$ further reduces to

$$
h\left(c_{L}, u \downarrow 1\right)=\varphi_{1}-4 \beta\left(1-\varphi_{1}\right) \varphi_{1}\left(1-c_{L}\right) c_{L}(u-1) .
$$

Equation (16) is the first order Taylor expansion of the packing fraction near $u=1$. So $\beta$ is a scaled proportionality contraction coefficient, governing the gradient of the binary void fraction $h$ at a size ratio $u$ of unity (when the packing is monosized, with void fraction $\varphi_{1}$ ) versus the size ratio $u$. Positive values of $\beta$ imply that the void fraction decreases (packing fraction increases) when some of the particles are larger (or smaller) relative to the others, so resulting in a void contraction. For these disordered packings it holds that, when particles of different sizes are combined, the void fraction is reduced (positive contraction) [19-23]. For ordered (crystalline) packings $\beta$ is negative, featuring a void fraction increase (packing fraction reduction) when polydispersity is introduced in these packing types. Also here $\beta$ is a function of $\varphi_{1}: \beta=-\frac{3}{4} \varphi_{1}^{-1}[21]$.

In Fig. 2 the scaled binary void fraction, based on Eq. (15), for the random close packing of spheres is set out versus the volume fraction of the large constituent $c_{L}$ for $u=2$. For this particle shape and densification it holds that $\varphi_{1}=0.36$, $\beta=0.20[7,19,21,23]$. In [21] this expression was extensively validated using experimental values, as well as results from computer generated sphere packings, taken from the literature. In Fig. 2 more recent simulation results concerning binary RCP of spheres are included [29]. Again good agreement between analytical Eq. (15) and the computer generated values can be observed. Note that the binary void fraction for small $u-1$ is fully characterized by $\varphi_{1}$ and $\beta$, which are only particle shape and compaction state dependent.

\section{B. Analysis of the contraction coefficient}

For the scaled random binary packing, e.g., as depicted in Fig. 2, by definition the void fraction is located within the shaded area enclosed by Eqs. (13) and (14) (pertaining to $u \rightarrow \infty$ ), and the line $h / \varphi_{1}=1$ (pertaining to monosized case, $u=1$ ), and depends on the composition $c_{L}$ and the size ratio $u$. Equations (13) and (14) reveal that maximum void reduction takes place for $u \rightarrow \infty$, at a composition $c_{L}=\left(1+\varphi_{1}\right)^{-1}$, which is indicated in the graph, and the scaled void reduction $h / \varphi_{1}$ then takes the minimum value $\varphi_{1}$.

Now, one would tentatively expect that in the entire area enclosed by Eqs. (13) and (14), and the line $h / \varphi_{1}=1$, hence

\begin{tabular}{|c|c|c|c|c|c|c|c|}
\hline & Material & Packing & Shape & $f_{1}$ & $\varphi_{1}$ & $\beta$ & $f_{1} \beta / \varphi_{1}$ \\
\hline \multirow[t]{9}{*}{ (a) } & Steel or simulation & $\mathrm{RCP}$ & Spherical & 0.638 & 0.362 & 0.20 & 0.352 \\
\hline & Sand (OS) & $\mathrm{RCP}$ & $\Psi=0.86$ & 0.624 & 0.376 & 0.25 & 0.415 \\
\hline & Sand (MR) & $\mathrm{RCP}$ & $\Psi=0.81$ & 0.574 & 0.426 & 0.26 & 0.350 \\
\hline & Quartz & $\mathrm{RCP}$ & Fairly angular & 0.503 & 0.497 & 0.373 & 0.378 \\
\hline & Feldspar & $\mathrm{RCP}$ & Plate-shaped & 0.497 & 0.503 & 0.374 & 0.370 \\
\hline & Dolomite & $\mathrm{RCP}$ & Fairly rounded & 0.495 & 0.505 & 0.347 & 0.340 \\
\hline & Sillimanite & $\mathrm{RCP}$ & Distinctly angular & 0.469 & 0.531 & 0.395 & 0.349 \\
\hline & Simulation & RLP & Spherical & 0.55 & 0.45 & 0.16 & 0.196 \\
\hline & Plastic & RLP & Cubical & 0.567 & 0.433 & 0.13 & 0.175 \\
\hline \multirow[t]{6}{*}{ (b) } & Simulation & $\mathrm{RCP}$ & $L / d=0, \Psi=1$ & 0.645 & 0.355 & 0.206 & 0.374 \\
\hline & Simulation & $\mathrm{RCP}$ & $L / d=0.1, \Psi=0.998$ & 0.672 & 0.328 & 0.149 & 0.305 \\
\hline & Simulation & $\mathrm{RCP}$ & $L / d=0.35, \Psi=0.981$ & 0.686 & 0.314 & 0.146 & 0.319 \\
\hline & Simulation & $\mathrm{RCP}$ & $L / d=1 \Psi=0.921$ & 0.659 & 0.341 & 0.207 & 0.400 \\
\hline & Simulation & $\mathrm{RCP}$ & $L / d=1.5, \Psi=0.878$ & 0.645 & 0.355 & 0.199 & 0.362 \\
\hline & Simulation & $\mathrm{RCP}$ & $L / d=2, \Psi=0.840$ & 0.630 & 0.370 & 0.190 & 0.324 \\
\hline
\end{tabular}

TABLE II. (a) Simulated and experimental values for monosized void (packing) fractions $\varphi_{1}\left(f_{1}\right)$, and the contraction coefficient $\beta$, for various particle shapes and their mode of packing [19,23]; $\Psi$ is the Wadell sphericity [Eq. (19)]. (b) Simulated values for monosized void (packing) fractions $\varphi_{1}\left(f_{1}\right)$ and $\beta$, for random close packing (RCP) of spherocylinders with different aspect ratios $L / d$ [29]; $\Psi$ is the Wadell sphericity [Eq. (20)]. 
also in the vicinity of $u=1$, the void reduction scales with $\varphi_{1}$ :

$$
\frac{h}{\varphi_{1}}=1-4 B \varphi_{1}\left(1-c_{L}\right) c_{L}(u-1),
$$

with $B$ as proportionality constant, which is a more general contraction coefficient, depending on the state of compaction only. This ansatz implies that $\beta\left(1-\varphi_{1}\right)$ is linearly dependent on $\varphi_{1}$; see Eq. (16):

$$
\beta\left(1-\varphi_{1}\right)=B \varphi_{1} .
$$

This conjectured relation, following from the foregoing reasoning, is verified by considering known values of $\beta$ for a number of particle shapes and compaction states, in particular RCP and RLP, of which information on void reduction is available. From simulations and experiments, for a number of particle shapes and packing types (random close packing and random loose packing) values of $\beta$ can namely be extracted, summarized in Table II(a).

In Table II(a), $\beta\left(1-\varphi_{1}\right)$ is divided by $\varphi_{1}$, and for the randomly close packed particles a relatively constant value of about 0.36 is obtained, confirming Eq. (18). In order to obtain $\beta$ values for more particle types, the binary packing results of spherocylinders with various $L / d$ ratios $(0,0.1$, $0.35,1,1.5$, and 2) of [29] are analyzed. Their computed values for $L / d=0$ (spheres) were already depicted in Fig. 2. The resulting $\left(1-\varphi_{1}\right)$ and $\beta$ are included in Table II(b). The computed monosized packing fractions of the spherocylinders by [29] are compatible with those reported in [30-33].

For the particles listed in Table I, also the Wadell sphericity [34] is computed, defined as

$$
\Psi=\frac{\pi^{1 / 3}\left(6 V_{P}\right)^{2 / 3}}{A_{P}} .
$$

The sphericity $\Psi$ of a particle is the ratio of the surface area of a sphere (with the same volume as the given particle) to the surface area of the particle. For the mentioned spherocylinders the sphericity follows as

$$
\Psi=\frac{\left(1+\frac{3 L}{2 d}\right)^{2 / 3}}{\left(1+\frac{L}{d}\right)} .
$$

The sphericity is highest for $L / d=0$ (spheres), for which $\Psi$ is unity, and it decreases monotonically with increasing $L / d$.

In Fig. 3 all values of $\beta^{\mathrm{RCP}}\left(1-\varphi_{1}^{\mathrm{RCP}}\right)$ of Table II, concerning $\mathrm{RCP}$, are set out versus $\varphi_{1}^{\mathrm{RCP}}$, including Eq. (18), the best linear fit yielding $B^{\mathrm{RCP}}=0.358$ with an $R^{2}$ of more than 0.99 . This fit not only confirms the conjecture, Eq. (18), this expression enables the prediction of $\beta^{\mathrm{RCP}}$ when the RCP monosized void fraction $\varphi_{1}$ of a particle shape is known. Note that both $\varphi_{1}$ and $\beta$ are physically defined properties, properties that depend on the particle shape and densification only, and which are uniquely related by Eq. (18). So, likewise the situation for an infinitely large size ratio, also for small $u-1$ it appears that the scaled void reduction apparently scales with $\varphi_{1}$ (Fig. 2). Actually one might expect that for all $u$ and $c_{L}$ the scaled binary void fraction, that are all captured in the previously discussed enclosed area in Fig. 2, the void fraction scales with $\varphi_{1}$.

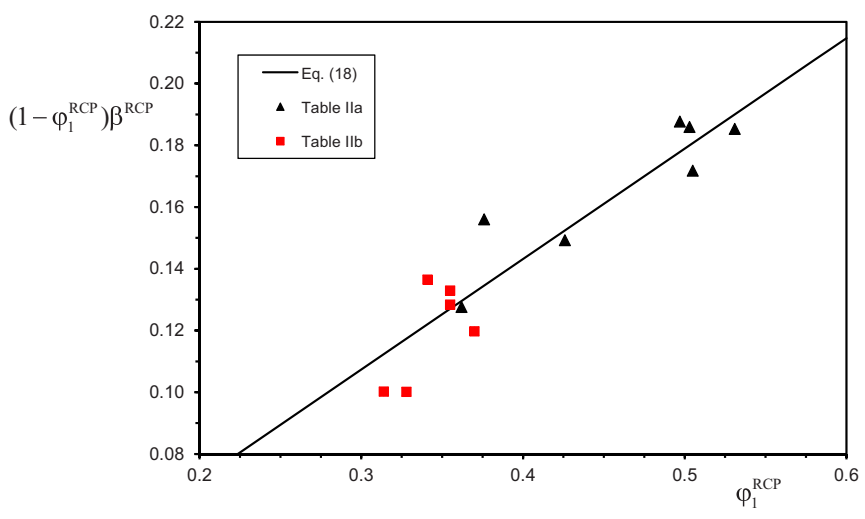

FIG. 3. Values of $\left(1-\varphi_{1}^{\mathrm{RCP}}\right) \beta^{\mathrm{RCP}}$ versus $\varphi_{1}^{\mathrm{RCP}}$ for a number of particle shapes (Table II), and Eq. (18) with $B^{\mathrm{RCP}}=0.358$.

The scaled binary void fraction graph of RCP, presented in Fig. 2, can also be constructed for RLP, and all intermediate states of compaction (with other values for $\varphi_{1}$, obviously). The only requirement is that the binary packing undergoes the same compaction as the monosized small and large components, which then each also possess the same $\varphi_{1}$ (as the particles are similar). This consideration implies that Eqs. (17) and (18) should also hold for RLP. For packings other than RCP, however, fewer packing data, from which the contraction coefficient can be extracted, are available. Only the binary RLP of cubes and spheres are known to the author and are listed in Table II(a). Again it follows that $\beta^{\mathrm{RLP}}\left(1-\varphi_{1}^{\mathrm{RLP}}\right) / \varphi_{1}^{\mathrm{RLP}}$ is almost constant; hence these data support the conjecture that Eq. (18) holds for RLP as well, with an average $B^{\text {RLP }}=$ 0.185 [Table II(a)]. Equation (18) with this $B^{\text {RLP }}$ enables the estimation of $\beta^{\mathrm{RLP}}$ when the RLP monosized void (or the packing fraction) $\varphi_{1}^{\mathrm{RLP}}$ of a given particle shape is known.

The main conclusion of this section is that, by invoking Eq. (18), the void fractions of the lognormal and Weibull particle size distributions, viz. Eqs. (11) and (12), can be written as

$$
\varphi^{\mathrm{LN}}=\varphi_{1} e^{-\sqrt{2 \pi} \varphi_{1} B \ln \sigma_{g}}=\varphi_{1} \sigma_{g}^{-\sqrt{2 \pi} \varphi_{1} B}
$$

and

$$
\varphi^{\mathrm{WB}}=\varphi_{1} \mathrm{e}^{-2 \sqrt{\pi} \varphi_{1} B / m},
$$

respectively. Whereas contraction coefficient $\beta$ in Eqs. (11) and (12), likewise $\varphi_{1}$, depends on both particle shape (type) and the compaction state, general contraction coefficient $B$ depends on the latter only. Its value ranges from 0.185 (RLP) to 0.358 (RCP). In Eqs. (21) and (22), the effect of particle shape and densification on void fraction is accounted for by the monosized void fraction $\varphi_{1}$. In both equations the monosized void fraction is found in both coefficients appearing on the right-hand sides. One can see that for particles with a larger monosized void fraction, its void reduction by polydispersity is more pronounced as the second coefficient, the exponential function, of expressions (21) and (22), will then deviate more from unity. Applying less compaction to obtain a looser packing and achieve a larger monosized void fraction is, however, counterproductive, as then also a smaller $B$ applies. This effect dominates the product $\varphi_{1} B$; in the Appendix it is derived that $B$ is linearly proportional to $\left(1-\varphi_{1}\right) / \varphi_{1}^{2}$. 

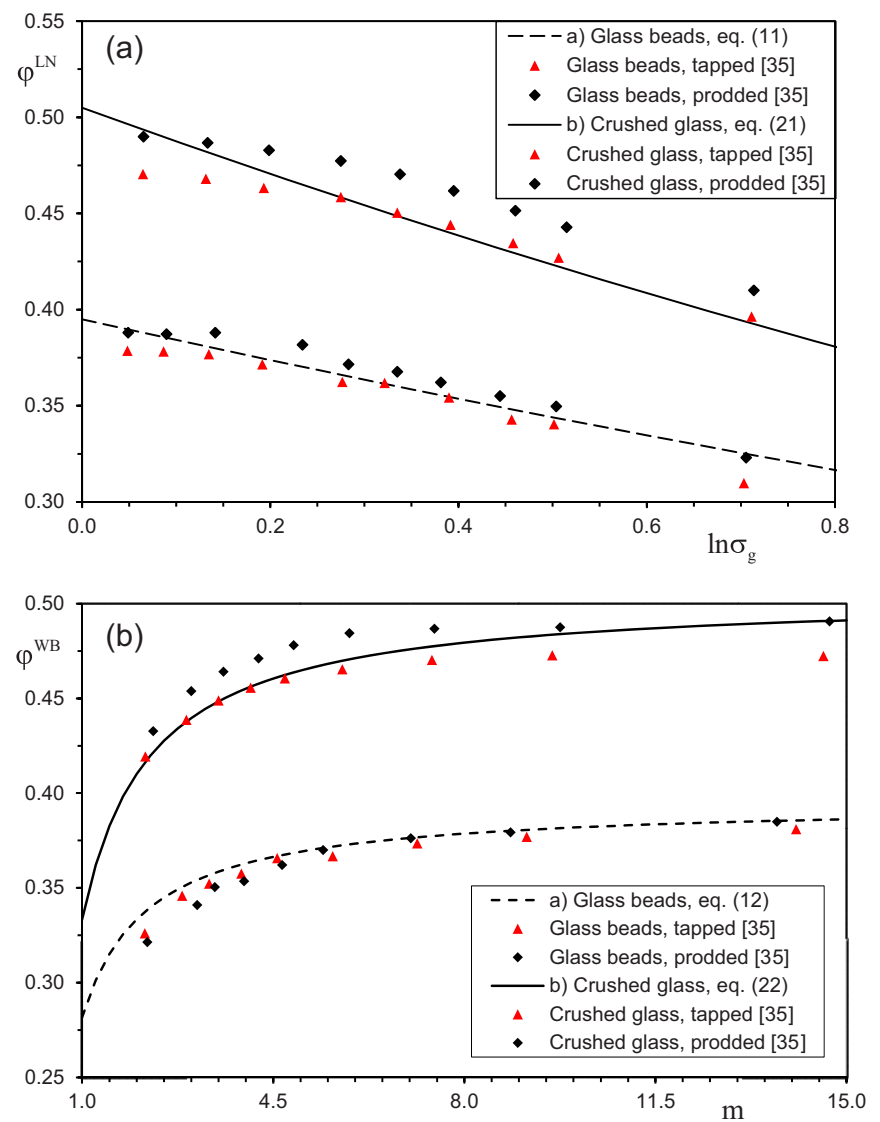

FIG. 4. (a) Experimentally measured [35] void fraction of glass beads and crushed glass particles with various lognormal size distributions, using tapping and prodding, versus the standard deviation $\ln \sigma_{g}$. (a) Equation (11) with $\varphi_{1}=0.395$ and $\beta=0.182$ (glass beads), and (b) Eq. (21) with $\varphi_{1}=0.505$ and $B=0.279$ (crushed glass). (b) Experimentally measured [35] void fraction of glass beads and crushed glass particles with various Weibull size distributions, using tapping and prodding, versus the distribution shape parameter $m$. (a) Equation (12) with $\varphi_{1}=0.395$ and $\beta=0.182$ (glass beads), and (b) Eq. (22) with $\varphi_{1}=0.505$ and $B=0.279$ (crushed glass).

\section{COMPARISON WITH REPORTED DATA}

A thorough verification of Eqs. (11), (12), (21), and (22), using the empirical data of Suzuki et al. [35], is presented here. To this end, the $\beta$ derived in the previous section is employed. Suzuki et al. [35] presented experimental measurements of multicomponent randomly packed beds with a number of distributions, among which are the lognormal and Weibull distributions. Glass beads (spherical particles) and crushed glass (irregularly shaped particles) were used for these experiments.

The measured void fractions of their packings following lognormal and Weibull size distributions are shown in Figs. 4(a) and 4(b), respectively. Their packings were compacted using tapping or prodding, which are indicated in Fig. 4. For all generated packings, either with glass beads or crushed glass, and for all lognormal and Weibull distributions, tapping always leads to a lower void fraction (better compaction) then for prodding. Figure 4 shows that for the beads the void fractions obtained with both compaction methods are closest, so one can conclude that an almost comparable compaction state was obtained using either method. For the crushed glass, on the other hand, one can see the difference in void fraction is more pronounced when tapping or prodding was employed.

From the void fraction values for small $\ln \sigma_{g}$ [Fig. 4(a)] and large $m$ [Fig. 4(b)], one can conclude that the monosized packing fraction $\left(\varphi_{1}\right)$ of the glass beads is 0.395 and for the crushed glass it amounts to about 0.505 . The void fraction 0.395 of the glass beads implies a state of packing between $\operatorname{RLP}\left(\varphi_{1}^{\mathrm{RLP}} \approx 0.46\right)$ and $\operatorname{RCP}\left(\varphi_{1}^{\mathrm{RCP}} \approx 0.36\right)$. Particles can be packed between the loosest and closest states of densification, which does not only affect the monosized packing fraction, but also $\beta$. In [21] it was derived that for random packing the following relation between monosized void fraction $\varphi_{1}$ and contraction coefficient $\beta$ holds:

$$
\varphi_{1} \beta=\varphi_{1}^{\mathrm{RLP}} \beta^{\mathrm{RLP}}=\varphi_{1}^{\mathrm{RCP}} \beta^{\mathrm{RCP}} .
$$

Equation (23) is a unique relationship between the contraction coefficient pertaining to a particle shape, and the state of compaction of such particle, where $\varphi_{1}$ ranges from $\varphi_{1}^{\mathrm{RCP}}$ to $\varphi_{1}^{\mathrm{RLP}}$ when the state of packing ranges from RCP to RLP $[21,23]$. Using $\varphi_{1}^{\mathrm{RCP}}=0.36$ and $\beta^{\mathrm{RCP}}=0.20$ of the spheres, and $\varphi_{1}=0.395$ for the glass bead packing of [35], Eq. (23) yields that the pertaining $\beta$ of the beads packing generated by [35] takes a value of 0.182 .

In Figs. 4(a) and 4(b), Eqs. (11) and (12) are drawn, respectively, using $\varphi_{1}=0.395$ and $\beta=0.182$, for $\ln \sigma_{g} \geqslant 0$ and $m \geqslant 1$, respectively. One can see that for all standard deviations $\ln \sigma_{g}$ and distribution shape parameters $m$, Eqs. (11) and (12) are able to well predict the packing density in the entire $\ln \sigma_{g}$ and $m$ ranges considered. To apply these closed-form expressions, no fitting parameters were needed. Furthermore, using the analysis of the previous section, the $B$ pertaining to the state of densification obtained by [35] for the tapped and prodded beads can be assessed. From Eq. (8), where $\beta=0.182$ and $\varphi_{1}=0.395$ for their glass bead assemblies, it follows that $B=0.279$, which is a value between $B^{\mathrm{RLP}}(0.185)$ and $B^{\mathrm{RCP}}(0.358)$ indeed.

For the crushed glass the measured data can be compared using Eqs. (21) and (22). We can assert that the state of packing is similar to that of the beads; both particle types were compacted in the same way, so that $B=0.279$ holds for beads and crushed glass alike. With this $B$, and $\varphi_{1}=0.505$ of the crushed glass, in Figs. 4(a) and 4(b), Eqs. (21) and (22) are drawn, respectively. For the entire range of standard deviations $\ln \sigma_{g}$ and shape parameters $m$, a fair agreement between Eqs. (21) and (22) and the empirical data can be observed.

In the Appendix it is shown that the monosized void fractions at different states of compaction are mathematically related by $B$, which is compaction dependent only. For the RLP and RCP void fractions of a number of particle types, the expression is compared with available data using the known values of $B^{\mathrm{RCP}}$ and $B^{\mathrm{RLP}}$, as derived in this paper.

\section{v. CONCLUSIONS}

In the present paper the void fraction of equally shaped polydisperse particles, with monosized void fraction $\varphi_{1}$ and having a Weibull (or Rosin-Rammler) size distribution, is 
addressed. As this distribution can be very similar to particles with a lognormal size distribution, for which a closed-form expression of the void fraction is known, first the parameters governing both distributions are mathematically related [Eqs. (8) and (10)]. Whereas the void fraction of the lognormal (or log-normal) distribution is governed by the standard deviation $\ln \sigma_{g}$, for the Weibull distribution it is determined by its distribution shape parameter $m$. To find a relation between both distributions, the ratios of the sizes at the $100 x$ th percentile and the $100(1-x)$-th percentile are matched, yielding a linear relation [Eq. (8)]. The subsequent coupling of $\ln \sigma_{g}$ and $m$ enables the application of the lognormal void fraction to Weibull size distributions [37].

The primary factors controlling the Weibull void fraction are the monosized void fraction $\varphi_{1}$, the shape parameter $m$ of the distribution, and the contraction parameter $\beta$. This latter property governs the contraction of disordered packings when the polydispersity increases. Likewise the unimodal void fraction, $\varphi_{1}, \beta$ is a nonadjustable property that is governed by particle shape and state of compaction only. For a number of particle shapes and RCP and RLP compaction states, its value is extracted from computer simulations and experiments and their values are summarized in Table II. The positive magnitude of $\beta$ is a measure for the packing fraction increase (or void fraction decrease) as the arrangement becomes more polydisperse, as is the case with the continuous Weibull size distributions with smaller $m$, and which was also seen with lognormal size distributions with increasing $\ln \sigma_{g}$ [23].

Subsequently, the $\beta$ of different particle shapes are analyzed. A consideration of the scaled binary void fraction (Fig. 2), reflecting the effect of combining binary particles on void reduction, yields the conjecture that $\beta\left(1-\varphi_{1}\right)$ is linearly proportional with $\varphi_{1}$ (Fig. 3). Available data on random close packing (RCP) of a wide range of particle shapes confirm this linear relation, and a proportionality constant $B^{\mathrm{RCP}}=0.358$ is extracted. Also the limited data available from random loose packings (RLP) confirm the linear relation, for which it holds that $B^{\mathrm{RLP}}=0.185$. In other words, a unique algebraic expression relating $\beta$ to the monosized void fraction $\varphi_{1}$ is established. The analysis yields alternative expressions (17), (21), and (22), containing the general contraction coefficient $B$, which depends on the compaction state only (so not on particle shape). Its value ranges from approximately 0.185 (RLP) to 0.358 (RCP).

Using the established values of $\varphi_{1}$ and $\beta$ for spheres [19-23], the obtained closed-form expressions for the void fraction of lognormal and Weibull size distributions are found to be in good quantitative agreement with experimentally generated packing data. This comparison concerns a wide range of standard deviations $\ln \sigma_{g}$ and shape parameters $m$ (Fig. 4). This agreement is achieved by using foreknown values of $\varphi_{1}$ and $\beta$, without the need of any adjustment. Also, for irregularly shaped particles, crushed glass, satisfying agreement is obtained in the entire standard deviation $\ln \sigma_{g}$ and distribution shape parameter $m$ ranges (Fig. 4). For these particles $\beta$ was not known a priori. Here, the analysis of Sec. III is used, yielding $B$, and subsequently Eqs. (21) and (22) are applied.

Finally, in the Appendix a unique closed-form relation of a particle's monosized void fraction at different compaction

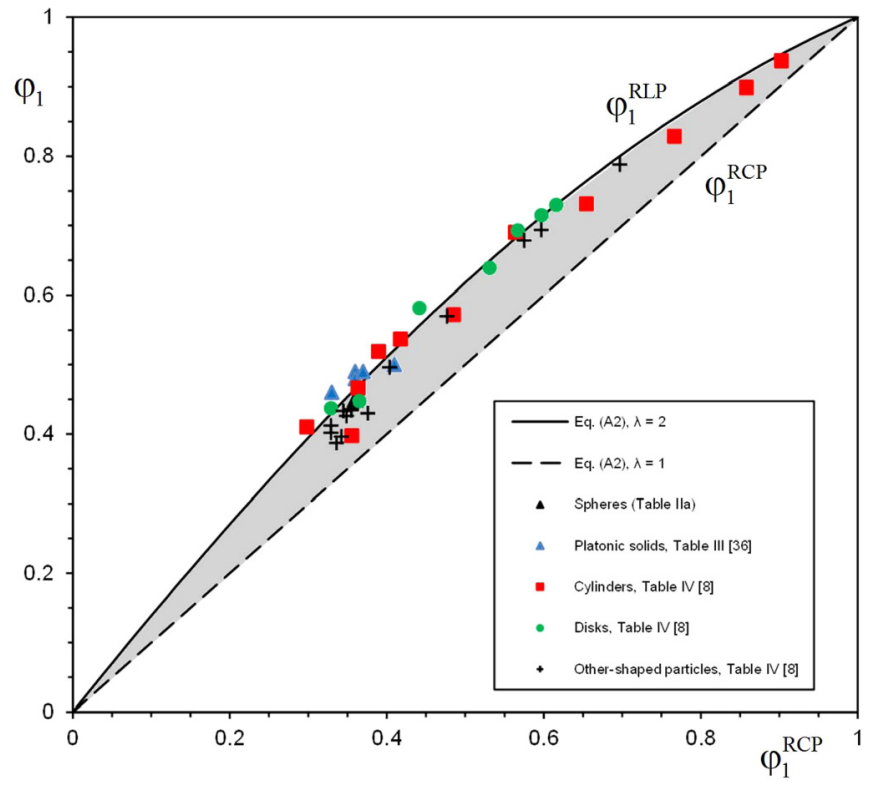

FIG. 5. Void fraction range of monosized particles as a function of $\varphi_{1}^{\mathrm{RCP}}$ (shaded area). The curve pertaining to the maximum void fraction, $\varphi_{1}^{\mathrm{RLP}}$, and the line pertaining to the minimum void fraction, $\varphi_{1}^{\mathrm{RCP}}$, are shown using Eq. (A2) with $\lambda=2$ and $\lambda=1$, respectively, as well as known combinations of $\varphi_{1}^{\mathrm{RLP}}$ and $\varphi_{1}^{\mathrm{RCP}}$ values for a number of particle shapes.

states [Eq. (A2)] is derived, with $B$ as sole parameter. This expression is applied to monosized loose and close packing data of many different particle types, using $B^{\mathrm{RLP}}=0.185$ and $B^{\mathrm{RCP}}=0.358$ [yielding $\lambda \approx 2$, Eq. (A3)], and good agreement is obtained (Fig. 5).

\section{APPENDIX}

Equation (18), which holds for all states of densification (from RLP to RCP), and Eq. (23) can be combined, yielding a closed-form relation between $\varphi_{1}, \varphi_{1}^{\mathrm{RCP}}$, or $\varphi_{1}^{\mathrm{RLP}}$ and their pertaining value of $B$ :

$$
B \frac{\left(\varphi_{1}\right)^{2}}{\left(1-\varphi_{1}\right)}=B^{\mathrm{RCP}} \frac{\left(\varphi_{1}^{\mathrm{RCP}}\right)^{2}}{\left(1-\varphi_{1}^{\mathrm{RCP}}\right)}=B^{\mathrm{RLP}} \frac{\left(\varphi_{1}^{\mathrm{RLP}}\right)^{2}}{\left(1-\varphi_{1}^{\mathrm{RLP}}\right)}
$$

So, when the $B$ pertaining to a certain state of compaction is known, with $B^{\mathrm{RCP}}=0.358$ (or $B^{\mathrm{RLP}}=0.185$ ) and a given $\varphi_{1}^{\mathrm{RCP}}$ (or $\varphi_{1}^{\mathrm{RLP}}$ ), the $\varphi_{1}$ pertaining to that compaction state can be computed. Or alternatively, as was the case in Sec. IV, with known $\varphi_{1}, B^{\mathrm{RCP}}$, and $\varphi_{1}^{\mathrm{RCP}}$ of spheres, $B$ pertaining to the compaction state of this measured $\varphi_{1}$ was assessed, and this $B$ was then used for the void fraction computation of the crushed glass.

Rewriting Eq. (A1) and solving the resulting quadratic expression yields $\varphi_{1}$ as a function of $\varphi_{1}^{\mathrm{RCP}}$ :

$$
\begin{aligned}
& \varphi_{1}\left(\varphi_{1}^{\mathrm{RCP}}, \lambda\right) \\
& =\varphi_{1}^{\mathrm{RCP}}\left\{\frac{-\lambda \varphi_{1}^{\mathrm{RCP}}+\left[\left(\lambda \varphi_{1}^{\mathrm{RCP}}\right)^{2}+4 \lambda\left(1-\varphi_{1}^{\mathrm{RCP}}\right)\right]^{1 / 2}}{2\left(1-\varphi_{1}^{\mathrm{RCP}}\right)}\right\},
\end{aligned}
$$


TABLE III. Random close and loose void fractions of five platonic solids as measured by [36].

\begin{tabular}{lll}
\hline \hline Shape & $\varphi_{1}^{\mathrm{RCP}}$ & $\varphi_{1}^{\mathrm{RLP}}$ \\
\hline Tetrahedron & 0.36 & 0.49 \\
Cube & 0.33 & 0.46 \\
Octahedron & 0.36 & 0.48 \\
Dodecahedron & 0.37 & 0.49 \\
Icosahedron & 0.41 & 0.50 \\
\hline \hline
\end{tabular}

with

$$
\lambda=\frac{B^{\mathrm{RCP}}}{B} .
$$

Alternatively, $\varphi_{1}$ can also be expressed as a function of $\varphi_{1}^{\mathrm{RLP}}$, yielding Eqs. (A2) and (A3) with $\varphi_{1}^{\mathrm{RCP}}$ and $B^{\mathrm{RCP}}$ replaced by $\varphi_{1}^{\mathrm{RLP}}$ and $B^{\mathrm{RLP}}$, respectively.

Values of $B^{\mathrm{RCP}}$ and $B^{\mathrm{RLP}}$ were derived in the foregoing, so for RLP and RCP this equation can be validated for particles with known void fractions at these two packing states. In Fig. 5, $\varphi_{1}^{\mathrm{RLP}}$ is set out versus $\varphi_{1}^{\mathrm{RCP}}$, based on Eq. (A2) and using $\lambda=2$, as $B^{\mathrm{RCP}} / B^{\mathrm{RLP}} \approx 2$ (Sec. III); see Eq. (A3). It is required that $\varphi_{1}^{\mathrm{RLP}}$ and $\varphi_{1}^{\mathrm{RCP}}$ coincide when $\varphi_{1}^{\mathrm{RLP}}$ and $\varphi_{1}^{\mathrm{RCP}}$ approach zero and unity, respectively, since $0 \leqslant \varphi_{1}^{\mathrm{RCP}} \leqslant \varphi_{1}^{\mathrm{RLP}} \leqslant 1$. This condition is fulfilled by Eq. (A2) indeed. Furthermore, the maximum absolute difference between $\varphi_{1}^{\mathrm{RLP}}$ and $\varphi_{1}^{\mathrm{RCP}}$ takes place at $\varphi_{1}^{\mathrm{RCP}}=0.525$ and $\varphi_{1}^{\mathrm{RLP}}=0.643$.

In this figure also the known $\varphi_{1}^{\mathrm{RCP}}$ and $\varphi_{1}^{\mathrm{RLP}}$ of spheres is included [Table II(a)]. In Table III the RLP and RCP void fractions of the five platonic solids, taken from [36], are listed, and included in Fig. 5 as well. These authors experimentally determined the RLP and RCP packing fractions of the monosized five platonic solids: tetrahedron, cube, octahedron, dodecahedron, and icosahedron, all having slightly rounded edges. Table III reveals that the RLP fraction of their rounded cubes amounts to 0.54 , which is compatible with the cubes packing fraction of 0.567 , listed in Table II(a).

Furthermore, for a number of disks, cylinders, and othershaped particles, the RLP and RCP void fractions were measured by [8], depicted in their Figs. 2(a) and 2(b), respectively, versus the Wadell sphericity. Hence, for each particle, $\varphi_{1}^{\mathrm{RLP}}$ and $\varphi_{1}^{\mathrm{RCP}}$ can be taken from their Figs. 2(a) and 2(b) [8], respectively, which are summarized in Table IV, and they are included in Fig. 5 as well.

Comparing Eq. (A2), invoking $\lambda=2$, with the empirical data which are plotted in Fig. 5 yields that there is reasonable to good agreement. Furthermore, notwithstanding that the void fraction of different particle types with identical $\Psi$ may differ
TABLE IV. Measured RCP and RLP void fraction values for different particle types and their Wadell sphericity values, taken from Fig. 2 of [8].

\begin{tabular}{|c|c|c|c|c|c|c|c|c|}
\hline \multicolumn{3}{|c|}{ Cylinders } & \multicolumn{3}{|c|}{ Disks } & \multicolumn{3}{|c|}{ Other-shaped particles } \\
\hline$\Psi$ & $\varphi_{1}^{\mathrm{RCP}}$ & $\varphi_{1}^{\mathrm{RLP}}$ & $\Psi$ & $\varphi_{1}^{\mathrm{RCP}}$ & $\varphi_{1}^{\mathrm{RLP}}$ & $\Psi$ & $\varphi_{1}^{\mathrm{RCP}}$ & $\varphi_{1}^{\mathrm{RLP}}$ \\
\hline 1 & 0.356 & 0.397 & 0.608 & 0.329 & 0.437 & 0.862 & 0.336 & 0.387 \\
\hline 0.872 & 0.298 & 0.410 & 0.532 & 0.365 & 0.447 & 0.821 & 0.329 & 0.402 \\
\hline 0.740 & 0.364 & 0.466 & 0.356 & 0.442 & 0.582 & 0.801 & 0.342 & 0.396 \\
\hline 0.678 & 0.390 & 0.519 & 0.203 & 0.531 & 0.639 & 0.793 & 0.329 & 0.412 \\
\hline 0.622 & 0.418 & 0.537 & 0.173 & 0.567 & 0.693 & 0.769 & 0.376 & 0.430 \\
\hline 0.562 & 0.485 & 0.572 & 0.138 & 0.597 & 0.715 & 0.737 & 0.355 & 0.434 \\
\hline 0.510 & 0.564 & 0.690 & 0.092 & 0.616 & 0.730 & 0.723 & 0.355 & 0.436 \\
\hline 0.478 & 0.655 & 0.731 & & & & 0.684 & 0.345 & 0.433 \\
\hline 0.409 & 0.766 & 0.829 & & & & 0.568 & 0.404 & 0.496 \\
\hline 0.370 & 0.858 & 0.899 & & & & 0.554 & 0.349 & 0.426 \\
\hline \multirow[t]{4}{*}{0.329} & 0.903 & 0.937 & & & & 0.450 & 0.597 & 0.693 \\
\hline & & & & & & 0.423 & 0.477 & 0.570 \\
\hline & & & & & & 0.413 & 0.697 & 0.788 \\
\hline & & & & & & 0.285 & 0.575 & 0.678 \\
\hline
\end{tabular}

(see Fig. 2 in [8] and Table IV), $\varphi_{1}^{\mathrm{RCP}}$ and $\varphi_{1}^{\mathrm{RLP}}$ seem to obey Eq. (A2). This agreement, in turn, indirectly supports the validity of underlying Eqs. (18) and (23), and the proposed values of $B^{\mathrm{RCP}}$ and $B^{\mathrm{RLP}}$ in Sec. III (leading to their ratio of approximately 2 ).

In this Appendix, Eqs. (18) and (23) have been combined to relate monosized random void fractions to the monosized void fraction of RCP, resulting in Eq. (A2). This equation has been drawn for RLP, so $\lambda=2$. For intermediate states of compaction, for which $1 \leqslant \lambda \leqslant 2$ as $B^{\mathrm{RLP}} \leqslant B \leqslant B^{\mathrm{RCP}}$ [see Eq. (A3)], Eq. (A2) is located in the shaded area of Fig. 5. The upper bound is the curve pertaining to the RLP void fraction, which is the curve drawn using Eq. (A2) with $\lambda=2$. The lower void fraction bound is the RCP void fraction, which follows from Eq. (A2) with $\lambda=1$ [so when $B$ equals $B^{\mathrm{RCP}}$, Eq. (A3)], Eq. (A2) then yields the straight line $\varphi_{1}=\varphi_{1}^{\mathrm{RCP}}$.

\section{ACKNOWLEDGMENTS}

The author wishes to thank Dr. R. Yu, M.Sc. for the assistance with the retrieving of the data of [29] and [35], presented in Figs. 2 and 4 and Table II(b), and Mrs. V. Elfmarkova, Dipl. Eng. for the data extracted from [8], presented in Fig. 5 and Table IV.
[1] W. Weibull, J. Appl. Mech. 73, 293 (1951).

[2] P. Rosin and E. Rammler, J. Inst. Fuel 7, 29 (1933).

[3] G. Herdan and M. L. Smith, Small Particle Statistics (Elsevier, Amsterdam, 1953).

[4] H. F. W. Taylor, Cement Chemistry, 2nd ed. (Thomas Telford, London, 1997).
[5] C. F. Moukarzel, S. F. Fernández-Sabido, and J. C. Ruiz-Suárez, Phys. Rev. E. 75, 061127 (2007).

[6] S. Levy, J. F. Molinari, I. Vicari, and A. C. Davison, Phys. Rev. E. 82, 066105 (2010).

[7] G. D. Scott, Nature (London) 188, 908 (1960); G. D. Scott and D. M. Kilgour, Br. J. Appl. Phys. 2, 863 (1969). 
[8] R. P. Zou and A. B. Yu, Powder Technol. 88, 71 (1996).

[9] S. Torquato, T. M. Truskett, and P. G. Debenedetti, Phys. Rev. Lett. 84, 2064 (2000).

[10] Y. Jiao, F. H. Stillinger, and S. Torquato, Phys. Rev. E 81, 041304 (2010).

[11] C. E. Zachary, Y. Jiao, and S. Torquato, Phys. Rev. E 83, 051309 (2011).

[12] Y. Jiao and S. Torquato, Phys. Rev. E 84, 041309 (2011).

[13] D. Chen, Y. Jiao, and S. Torquato, J. Phys. Chem. B 118, 7981 (2014).

[14] G. Y. Onoda and E. G. Liniger, Phys. Rev. Lett. 64, 2727 (1990).

[15] K. J. Dong, R. Y. Yang, R. P. Zou, and A. B. Yu, Phys. Rev. Lett. 96, 145505 (2006)

[16] M. Jerkins, M. Schröter, H. L. Swinney, T. J. Senden, M. Saadatfar, and T. Aste, Phys. Rev. Lett. 101, 018301 (2008).

[17] C. Song, P. Wang, and H. A. Makse, Nature 453, 629 (2008).

[18] G. W. Delaney, T. Di Matteo, and T. Aste, Soft Matter 6, 2992 (2010).

[19] H. J. H. Brouwers, Phys. Rev. E 74, 031309 (2006); 74, 069901 (2006); 84, 059905 (2011).

[20] H. J. H. Brouwers, Phys. Rev. E 84, 042301 (2011).

[21] H. J. H. Brouwers, Phys. Rev. E 87, 032202 (2013).

[22] H. J. H. Brouwers, Phys. Rev. E 88, 032204 (2013).

[23] H. J. H. Brouwers, Phys. Rev. E 89, 052211 (2014).
[24] C. C. Furnas, Report of Investigations, Serial No. 2894 (U. S. Department of Commerce, Bureau of Mines, Washington, D.C, 1928); Bull. - U. S., Bur. Mines 307, 74 (1929).

[25] C. C. Furnas, Ind. Eng. Chem. 23, 1052 (1931).

[26] R. S. Farr and R. D. Groot, J. Chem. Phys. 131, 244104 (2009).

[27] A. V. Kyrylyuk, A. Wouterse, and A. P. Philipse, Prog. Colloid Polym. Sci. 137, 29 (2010).

[28] A. B. Hopkins, F. H. Stillinger, and S. Torquato, Phys. Rev. E 88, 022205 (2013).

[29] L. Y. Meng, P. Lu, S. X. Li, J. Zhao, and T. Li, Powder Technol. 228, 284 (2012).

[30] S. R. Williams and A. P. Philipse, Phys. Rev E 67, 051301 (2003).

[31] A. Wouterse, S. R. Williams, and A. P. Philipse, J. Phys.: Condens. Matter 19, 406215 (2007).

[32] A. Wouterse, Random packing of colloids and granular matter, Ph.D. Thesis, Utrecht University, 2008.

[33] J. Zhao, S. X. Li, R. P. Zou, and A. B. Yu, Soft Matter 8, 1003 (2012).

[34] H. Wadell, J. Geol. 43, 250 (1935).

[35] M. Suzuki, T. Oshima, H. Ichiba, and I. Hasegawa, KONA Powder Part. J. 4, 4 (1986); when the author prepared [23], he was not aware of the existence of this paper.

[36] J. Baker and A. Kudrolli, Phys. Rev. E 82, 061304 (2010).

[37] The packing fractions of lognormal and Weibull distributions read $1-\varphi^{\mathrm{LN}}$ and $1-\varphi^{\mathrm{WB}}$, respectively. 